\title{
Psychosocial assessment in the Emergency Department: The experiences of people presenting with self-harm and suicidality.
}

\author{
Penny Xanthopoulou, PhD (corresponding author). p.d.xanthopoulou@exeter.ac.uk \\ Mary Ryan, MD \\ Matthew Lomas, BSc \\ Rose McCabe, $\mathrm{PhD}$.
}

\begin{abstract}
Background: In the U.K, suicide risk is assessed in the emergency department (ED) in a face-to-face assessment with psychiatric liaison practitioners. This study aimed to explore patient experiences of psychosocial assessment after presenting with self-harm/suicidality. Method: Twenty-eight patients were interviewed within 2 weeks of ED attendance for selfharm/suicidality. Interviews were transcribed and analysed using inductive thematic analysis.

Results: People described two different experiences. A therapeutic interaction was about the 'person' and made people feel their life mattered and instilled hope for the future. This was characterised by: unscripted conversation; warmth promoting disclosure, psychological exploration of feelings; validation of distress; and a co-produced care plan. A formulaic assessment was about the 'risk' and made people feel their life did not matter and hopeless about the future. This was characterised by: feeling judged and not worthy of help, a focus on risk and form filling, a trivial treatment plan and loss of trust in services.

Limitations: A single ED; non-diverse sample.

Conclusion: Psychosocial assessment in the ED impacts on hope for people in crisis. A focus on therapeutic communication that is about the person, as well as the risk, improves patient experience, decreases distress and instils hope that life is worth living.
\end{abstract}

\section{Background}

Suicide is a major public health concern worldwide. In the UK, over 6,000 people died by suicide in 2018 (ONS, 2019). Self-harm is a strong predictor of suicide (House and Owens 2020; Chan et al., 2016) and refers to intentional self-poisoning or self-injury, irrespective of motive or the extent of suicidal intent (NICE, 2013). Each year, 220,000 episodes of selfharm are managed by emergency departments (EDs) in England. Suicidality tends to increase during socioeconomic crises and COVID-19 is expected to have a significant effect on people's mental health. This is expected to lead to an increase in self-harm and suicide rates worldwide (Gunnell et al., 2020). Around 40\% of people who have taken their lives have attended the ED in the year before their death (Da Cruz et al., 2011). The ED therefore is a crucial site for high quality care for people in a mental health crisis.

People who present to the ED with self-harm or suicidal ideation, undergo a psychosocial assessment by Liaison Psychiatry (LP) services, which are located in more than $90 \%$ of UK EDs (House and Owens, 2020; Chan et al., 2016). Around 60\% of people presenting to the ED with self-harm receive a psychosocial assessment (Kapur et al., 2008). NICE (2013) advise that self-harm in the ED should be treated with the same care, compassion and respect as any other condition, and the psychosocial assessment should address needs (e.g. coping strategies, physical problems); and risks (suicidal intent, risk) and protective factors. 
A limited number of studies have examined the ED experience from the patient perspective (Clarke et al., 2007; Thomas et al., 2018), with few focusing specifically on self-harm (Morphet et al., 2012; Bolger et al., 2004; MacDonald et al., 2004; MacDonald et al., 2020; Owens et al., 2016). They identify areas for improvement in the main ED environment, staff attitudes towards people who self-harm, and the need for a formal risk assessment. Other studies focus on patient experiences in inpatient psychiatric settings, highlighting the importance of respect and hope (Berglund et al., 2016; Vatne \& Nåden, 2014, Taylor et al., 2009). Only one study by Hunter et al. (2013) investigated experiences of the psychosocial assessment in the ED, with a small sample of 13 participants. They found that willingness to disclose distressing thoughts and plans is highly dependent on trust and the therapeutic relationship, and that patients need to be informed about the purpose of the psychosocial assessment for it to be meaningful for them (Hunter et al., 2013). In addition, self-stigma and stigma towards people who self-harm from practitioners (Pompili et al., 2005) make these conversations more challenging. The aim of this study was to explore the experiences of psychosocial assessment from the perspective of people attending the ED with self-harm and suicidality (suicidal thoughts and plans).

\section{Methods}

Data were collected as part of a larger mixed methods study, that video-recorded psychosocial assessments for self-harm/suicidality. The London-Central Research Ethics Committee gave ethical approval (17/LO/1234).

Patients referred by ED triage for a psychosocial assessment to $32 \mathrm{LP}$ practitioners $(76 \cdot 2 \%$ consented) in one LP service in England were eligible for inclusion. Practitioner roles were: mental health nurses, junior doctors, senior mental health practitioners, healthcare assistants and student nurses. Forty-five people (76 people approached, 59\% consented) participated in the larger study video-recording psychosocial assessments, of which 28 agreed to be interviewed. Participants were interviewed between 10/09/2018 to 09/04/2019, within 2 weeks of ED attendance. Participants were initially approached by a practitioner who assessed if they met the inclusion criteria: being over 16 years old with capacity to consent. Patients with cognitive difficulties, active psychosis, needing an interpreter, and those subject to a restriction order were excluded. If they had capacity, the practitioner asked for consent for a researcher to talk to them. A three-step informed consent process developed with people with lived experience was used (fig.1). 
Patient is seen by the triage nurse and then by a mental health practitioner within 1 hour of their referral, during which informed consent needs to be obtained Three-step consent process to compensate for the short period that
potential participants would have to decide whether or not to take part.

Written informed consent was obtained by the researcher prior to the psychosocial assessment. The researcher explained the study using an information sheet.

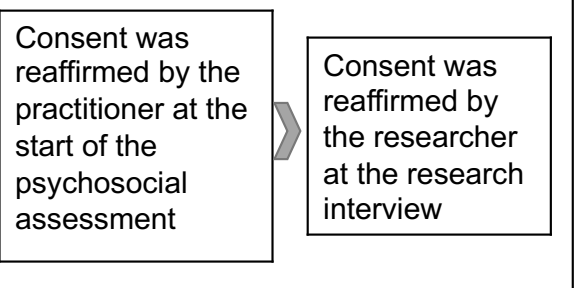

Figure 1. The informed consent process.

A semi-structured interview guide was used to elicit participants' experience of the ED and the LP psychosocial assessment (fig 2). The interview guide, consent forms and information sheets were developed with people with lived experience. Potential risk of causing distress when asking about sensitive issues was discussed with our lived experience group members during the study design. This were addressed by taking a sensitive approach to the interview, and a risk protocol was developed in the event of possible risk of suicide during the interview. Participants were offered a $£ 30$ voucher to thank them for participating.

\section{Psychosocial assessment}

- Can you tell me a bit about what questions the liaison team member asked?

- What did you think of the questions he/she asked?

o What did you think of the way he/she asked questions?

- How was the mental health professional as someone to talk to?

- How did you find talking about your self-harm/thoughts of ending your life?

- Did you get the opportunity to talk about things you wanted to?

o Were you able to answer openly?

- Is there anything you think could have improved the conversation?

- What do you consider the most important outcome of the meeting (or what was most helpful, if anything)?

o Was this what you expected?

- What do you think about the treatment plan?

- How did you feel during/after the conversation/ assessment?

- If you found yourself in a similar crisis again, what would you do?

Figure 2. Interview guide questions about the psychosocial assessment.

\section{Data analysis}

Interviews were audio recorded, transcribed verbatim and anonymised. Thematic analysis was used to identify patterns within data, by generating codes and identifying subthemes and overarching themes (Braun \& Clarke 2006). Transcripts were initially line-by-line coded by one author (PX) using NVivo12. Development of the coding scheme was iterative and involved initial familiarisation with the data, constant comparison of data and discussion of emerging codes by all authors. The codes were interrogated in 6 analytic meetings. The subthemes and themes were then finalised in six meetings involving authors PX, RM and 
MR. The funders had no role in the study design, data collection or data analysis/interpretation.

\section{Results}

Reasons for LP referral were suicidal ideation $(n=13)$ and self-harm $(n=15 ; 13$ overdose, 1 attempt to hang, 1 attempt to drown). Twenty-eight participants (table 1) were interviewed at their homes or via telephone 2-9 days after the assessment. Interviews lasted on average 22 minutes. For 15 patients this was their first psychosocial assessment at this hospital.

Eighteen participants were seen in ED and ten were treated in the short-stay Acute Medical Unit before being seen by LP, as they were admitted to be treated for self-harm before being assessed (see fig. 3).

\begin{tabular}{|l|l|l|}
\hline \multicolumn{3}{|l|}{ Table 1. Participants characteristics $(\mathbf{n = 2 8})$} \\
\hline Sex & Male & 9 \\
& Female & 19 \\
\hline Age & Mean & $35 \cdot 36$ \\
& Min & 18 \\
& Max & 76 \\
& SD & $16 \cdot 65$ \\
\hline Ethnicity & White & 27 \\
& Indian & 1 \\
\hline Employment & Studying & 7 \\
& Employed & 9 \\
& Retired & 3 \\
& Unemployed & 9 \\
\hline Education & School & 13 \\
& Further education & 13 \\
& Higher education & 2 \\
\hline
\end{tabular}

Table 1. Participant characteristics.

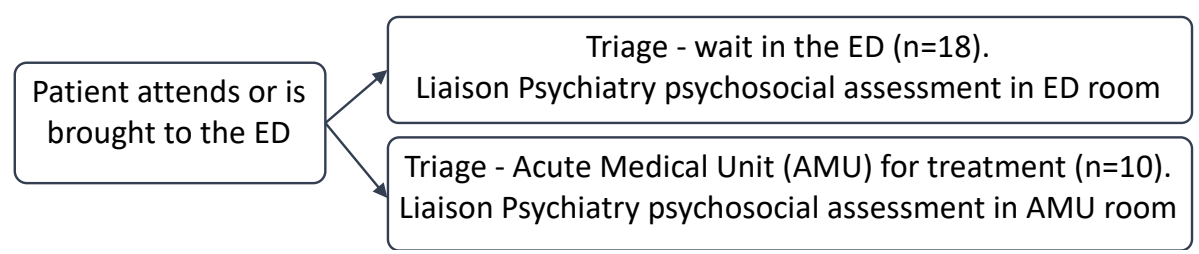

Figure 3. Participant journey.

We identified two main themes based on peoples' experiences (1) A therapeutic interaction made people feel their life mattered and instilled hope for the future. (2) A formulaic assessment focusing on risk made people feel their life did not matter and hopeless about the future (see table 2). Therapeutic conversations were characterised by: unscripted conversation, really listening and acknowledging distress; warmth, positive nonverbal communication that fosters trust and disclosure; difficult yet direct conversations helping people understand their feelings; and a co-produced treatment plan. Formulaic assessments were characterised by: checklist questions that are a barrier to trust, disclosure and listening; 
feeling judged and unworthy of help; trivial treatment suggestions; feeling unsafe to go home See also Figure 3 depicting participants' experiences of these two different approaches.

\begin{tabular}{|c|c|}
\hline Theme & Subthemes \\
\hline $\begin{array}{l}\text { Therapeutic conversations: } \\
\text { reducing distress and instilling } \\
\text { hope }\end{array}$ & $\begin{array}{l}\text { Unscripted conversation, listening and acknowledging distress } \\
\text { Explaining suicidality and positive nonverbal communication } \\
\text { Commitment to honest direct conversation } \\
\text { Relevant and co-produced treatment plan } \\
\text { Unique service in position to help and instil trust for future help- } \\
\text { seeking }\end{array}$ \\
\hline $\begin{array}{l}\text { Formulaic assessments: } \\
\text { focusing on risk }\end{array}$ & $\begin{array}{l}\text { Checklist questions: a barrier to trust and listening } \\
\text { Not taken seriously, feeling judged and not worthy of help } \\
>\quad \text { Physical and mental state: disoriented and hard to engage } \\
>\quad \text { Trivial treatment plan: long waits for overstretched services } \\
>\quad \text { Not feeling safe at discharge }\end{array}$ \\
\hline
\end{tabular}

Table 2: Overview of themes and subthemes.

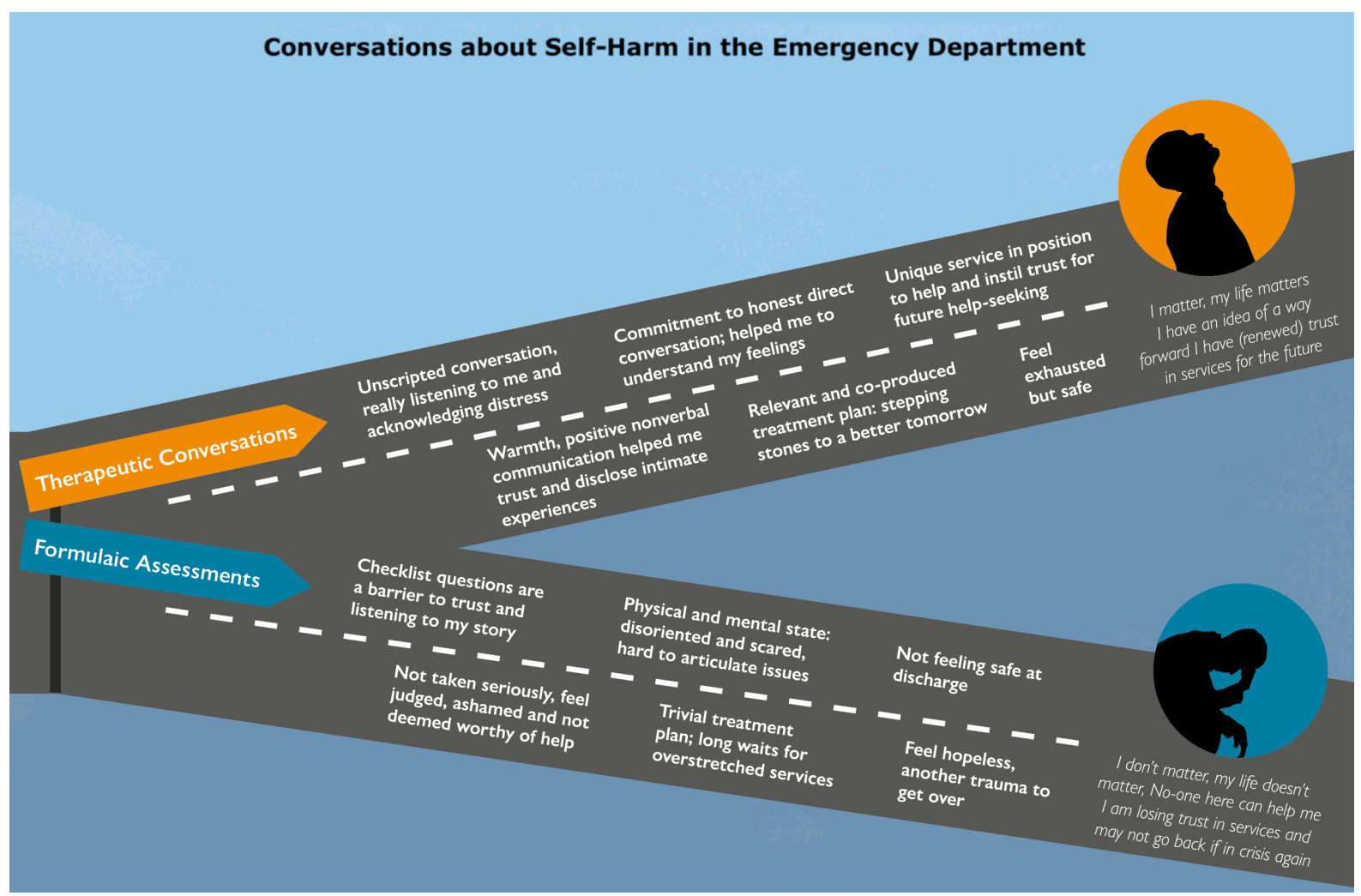

Figure 4. Psychosocial assessment in the ED: Therapeutic conversation vs formulaic assessment.

\section{THERAPEUTIC CONVERSATIONS: REDUCING DISTRESS AND INSTILLING HOPE}

\subsection{Unscripted conversation, listening and acknowledging distress}

Participants described a caring conversation that was not following a script: "felt a lot more like a conversation than an interrogation, which is probably one of the reasons it was a lot 
better than previously" (P24). Feeling listening to was described as being responsive and patient answers being followed up:

"They'd be taking the lead in the questions but depending on my answer they would decide how the conversation went, so it wasn't like a scripted thing... seemed to care a lot about me as a person rather than it just being their job" (P19).

People also highlighted being taken seriously, not feeling judged and having their suffering acknowledged. Being able to 'just talk' was an important outcome: "I knew that I could actually speak to somebody and that's why I have been a lot better since then” (P22).

\subsection{Explaining suicidality and positive nonverbal communication}

Participants found that a psychological exploration of feelings and exploring reasons for their suicidality was helpful: "What I was feeling and thinking. Helping me understand it, suicide and that" (P33). Normalising distress was also helpful, by offering a different perspective from which to consider and explain distress. Good eye contact and body language made people feel calm: “(Practitioners) were quite comfortable talking about stuff. They didn't tense up the body language” (P39).

\subsection{Commitment to honest direct conversation}

Many participants emphasised that they wanted help and trusted the practitioners: "I was there for help and I was putting my trust into them" (P22). Talking about suicidality was difficult, but people stressed their commitment to be truthful: "they're there to help so I tried to be as open and honest as possible" (P15). This helped to build a two-way trusting relationship: "Said ... 'I want to be honest with you'... and I was very honest back" (P07).

\subsection{Relevant and co-produced treatment plan}

At the end of their assessment, patients were given a care plan. This was described as helpful when it was relevant to one's needs with specific and practical solutions, and when it was coproduced so that people were able to engage with the plan: "The sleeping thing... and then the mindfulness thing is really ideas that I can work on" (P42). Follow up was also important: "My GP has been calling... was quite nice...I haven't really been followed-up before" (P35).

\subsection{Unique service in position to help and instil trust for future help-seeking}

Those unaware of the LP service expressed relief that it existed, as the LP was there to help in a time of crisis: "I needed help and they were there and they were in that position to help $m e$ " (P22). For some, this contrasted with previous negative experiences: "To some extent they restored my trust in the NHS mental health department" (P24).

\section{FORMULAIC ASSESSMENTS: FOCUSING ON RISK}

\subsection{Checklist questions: a barrier to trust and listening}

Many people described a formulaic style of questioning that was checklist-like, tedious and difficult to understand: "talking about self-harming... how many times I do it and I just lost track... I couldn't give him an answer... and I didn't really understand them” (P17). People talked about generic, closed questioning, giving them no opportunity to convey their thoughts or feelings. Faced with the aftermath of what happened, some people needed help in dealing with the impact on relationships, but they felt they had no opportunity to raise these concerns 
in the meeting: "my children are now asking me questions... how do I answer those questions for my children" (P02). Many felt this arose from a risk-focused agenda and felt pressured to answer these questions: "there was pressure on me to have an answer when I didn't ... it's actually all about risk and not about actually the person" (P16).

\subsection{Not taken seriously, feeling judged and not worthy of help}

People felt the burden of proof was on them to prove they were in serious distress. They described feeling negatively judged and stigmatised: "in those sorts of situations, people speak a lot down to me" (P35). Others tried to hide their distress for fear of being judged: "I was trying so hard to stay still and look happy, but inside I was literally crumbling... the nurses hate me in there, so I know just to shut up, smile" (P17).

After the psychosocial assessment, many were still struggling with ongoing suicidality and hopelessness, resigned to the reality of limited resources: "feeling quite hopeless - what more could be offered ... there's not going to be a sort of magical solution" (P37). People also struggled to understand their suicidality and felt guilt and regret: "I don't want to believe that I could have done that again to myself and to my family and to my children. But there's like a guilt I don't want to face up to" (P29).

\subsection{Physical and mental state: disoriented and hard to engage}

The mental and physical symptoms of suicidality posed an additional challenge for patients as they tried to navigate the ED process. Some people had their psychosocial assessment when they were not in a state to engage. For those who attended with a companion, they often spoke on their behalf: "my matron came with me ... and she did most of the talking there because... I still wasn't able to talk" (P16). Even when the interaction was positive it was difficult to absorb or remember what was said: "they were trying to say positive things, but in that space, it doesn't really touch you” (P16).

\subsection{Trivial treatment plan: long waits for overstretched services}

Participants were disappointed with their treatment plan when it involved generic information and leaflets/helplines: "Just gave me that phone number if I'm feeling really really low" (P01). In other cases, people were signposted to community services that had no capacity: "it's all very well kind of making suggestions, but if those suggestions aren't taken up by other people then there isn't anything that really came of it" (P37). Due to this many hadn't looked at their careplan, feeling it was redundant. People suggested direct referral to services would be better than having to initiate contact themselves.

\subsection{Not feeling safe at discharge}

Some people were surprised that they were discharged immediately after the assessment when they didn't feel safe: "they didn't listen as I told them I had a plan and also I wouldn't be able to keep myself safe... so it almost seems they don't care” (P30). People explained how although staying at the hospital was not their preferred option, it would have been safer for them: "I felt safe in that environment. And even though I wanted to come home even though it's a contradiction I felt quite scared. Because I can be a danger to myself" (P29). When the need for safety was not addressed many felt hopeless: "Now you go. And that's a bit...left in limbo" (P06). Despite this, some felt they had no other option but to go back if they found themselves in a similar situation: "I suppose I'd go through it again but it just feels like another trauma to get over" (P11). 


\section{Discussion}

Within a single ED, peoples' experiences of psychosocial assessments varied from a therapeutic conversation focusing on the person and instilling hope to a formulaic assessment focusing on risk and leaving the person feeling hopeless. A single time-limited encounter can have a positive impact for the person being assessed, when they were able to talk openly about their experiences, felt listened to and a care plan was co-produced with them. This decreased distress and instilled hope. Conversely, a risk-focused formulaic assessment had a negative impact. It can lead to people leaving the ED feeling their life doesn't matter, hopeless about a way forward and losing trust in whether they will ever be able to find help.

NICE (2013) describes psychosocial assessment as the assessment of needs and risks to understand and engage people who self-harm, and initiate a therapeutic relationship. Our findings highlight the importance of the relational aspects of these assessments, particularly the person telling their story and the practitioner carefully listening to them and validating their distress. When mental health practitioners acknowledge distress and show empathic nonverbal communication, this fosters trust and the disclosure of intimate experiences which provides clinically important information for a more valid assessment of risk. In line with other research (MacDonald et al., 2020), we found that these honest, if difficult, conversations, help people understand their suicidal feelings and contribute to a co-produced care-plan. Other studies also highlight the importance of a care-plan that is produced with the patient (Thomas et al., 2018; Hunter et al., 2013). Participants in our study reported a willingness to have difficult conversations and to be honest, because they wanted to be helped. Non-verbal communication was also important in demonstrating warmth and care, a factor that can often be overlooked (Philippot et al., 2003).

By contrast, people who described their experience negatively highlighted those elements in which their story and circumstances seemed of secondary importance in a more formulaic conversation. People found checklist-like questioning difficult to understand and felt under pressure to come up with the 'right' answer. They described how closed, leading questioning prevented openness and masked the complexity of the suicidal presentation. Others have also suggested that to explore the complexity of suicidality we need to look at patients' subjective experiences (Berglund et al., 2016). A formulaic approach appeared to have a negative impact on the perceived relevance or utility of the treatment plan. Recent evidence suggests that 'professionalism' in mental health care, defined by regulations, targets and measurements, has a negative impact on compassionate care (Bhui, 2016). Patients in our study felt there was a tension between practitioners' need to 'tick the right boxes' and a space to tell their story in a way that wasn't about coming up with the 'right answers' to tick the boxes. Our findings suggest that an assessment which begins with an unstructured conversation is more likely to lead to a fuller disclosure of thoughts and plans leading to a more valid risk assessment.

Positive communication has been shown to have a placebo effect in mental healthcare with benefits particularly linked to empathy, clarity of communication, non-verbal communication and involving the person in decision-making (Priebe et al., 2020). In contrast, formulaic assessments appear to engender nocebo or negative effects based on experiences, including lack of trust, negative expectations and negative framing of treatment (Benedetti et al., 2007). Patients' negative experiences of psychosocial assessments include not hearing the person's story (Hunter et al., 2013), not really listening, lack of warmth and empathy (Hunter et al., 
2013; Howick et al., 2018) and feeling negatively judged for seeking help (Vatne \& Nåden, 2014).

People who attend the ED frequently with suicidality/self-harm, in particular, report feeling judged by ED staff or feeling undeserving of help. Generalist ED staff report lacking the skills to assess mental health patients (Clarke et al., 2007). For patients, repeating cycles of crisis, where seeking help leads to a negative care experience, increases feelings of hopelessness which has a an increasing negative cumulative effect over time (Cully et al., 2020). This cycle of distress and despair is known to increase the risk of suicide (Vatne \& Nåden, 2014; Cully et al., 2020). The correlation between frequent attendance in the ED and increase in suicide is an important one (Crandall et al., 2006; Da Cruz et al., 2011). While fear of hospitalisation might lead to non-disclosure of suicidal thoughts and plans (Vatne \& Nåden, 2014), some patients in this study actively sought the safety of a hospital admission and felt unsafe to be discharged. In line with others, we also found that lack of privacy and long waits can intensify distress (Morphet et al., 2012; Clarke et al., 2007). Patients in our study describe a state of disorientation, feeling very emotional and exhausted, before, during and after the psychosocial assessment.

\section{Strengths and Limitations}

This study was conducted in a single ED with a non-diverse sample. Those who agreed to participate may not be representative of the wider patient group who present to the ED.

Practitioners with different professions/roles performed the assessments, which might impact on the way these were conducted. People with lived experience of suicidality/self-harm were closely involved in all stages of the study. We carried out interviews with a relatively large sample and soon after the psychosocial assessment limiting recall bias.

\section{Conclusion}

It is possible to improve the experience of people assessed by liaison psychiatry without many additional resources, by treating patients with compassion and offering hope. The patients in our study were helped most by being able to tell their story, being listened to and feeling cared for. A therapeutic conversation is not a luxury for people with suicidality and self-harm. It can make all the difference for people when their choice of living or dying hangs in the balance.

\section{Declaration of interests}

We declare no competing interests.

\section{Acknowledgments}

We thank all the patients, companions and healthcare practitioners who participated in the study.

\section{References}

Benedetti, F., Lanotte, M., Lopiano, L., \& Colloca, L. (2007). When words are painful: unraveling the mechanisms of the nocebo effect. Neuroscience, 147(2), 260-271.

https://doi.org/10.1016/j.neuroscience.2007.02.020 
Berglund, S., Åström, S., \& Lindgren, B. M. (2016). Patients' experiences after attempted suicide: A literature review. Issues in Mental Health Nursing, 37(10), 715-726.

https://doi.org/10.1080/01612840.2016.1192706

Bhui, K. S. (2016). Smuggling compassion into care: Is the NHS destined for system D?. Australian \& New Zealand Journal of Psychiatry, 50(7), 611-612.

https://doi.org/10.1177/0004867416654577

Bolger, S., O'Connor, P., Malone, K., \& Fitzpatrick, C. (2004). Adolescents with suicidal behaviour: attendance at A\&E and six month follow-up. Irish journal of psychological medicine, 21(3), 78-84. https://doi.org/10.1017/S0790966700008405

Braun, V., \& Clarke, V. (2006). Using thematic analysis in psychology. Qualitative research in psychology, 3(2), 77-101. 10.1191/1478088706qp063oa

Chan, M. K., Bhatti, H., Meader, N., Stockton, S., Evans, J., O'Connor, R. C., et al. (2016). Predicting suicide following self-harm: systematic review of risk factors and risk scales. The British Journal of Psychiatry, 209(4), 277-283. https://doi.org/10.1192/bjp.bp.115.170050

Clarke, D. E., Dusome, D., \& Hughes, L. (2007). Emergency department from the mental health client's perspective. International journal of mental health nursing, 16(2), 126-131. https://doi.org/10.1111/j.1447-0349.2007.00455.x

Crandall, C., Fullerton-Gleason, L., Aguero, R., \& LaValley, J. (2006). Subsequent suicide mortality among emergency department patients seen for suicidal behavior. Academic Emergency Medicine, 13(4), 435-442. https://doi.org/10.1197/j.aem.2005.11.072

Cully, G., Leahy, D., Shiely, F., \& Arensman, E. (2020). Patients' Experiences of Engagement with Healthcare Services Following a High-Risk Self-Harm Presentation to a Hospital Emergency Department: A Mixed Methods Study. Archives of suicide research, 121. https://doi.org/10.1080/13811118.2020.1779153

Da Cruz, D., Pearson, A., Saini, P., Miles, C., While, D., Swinson, N., et al. (2011). Emergency department contact prior to suicide in mental health patients. Emergency Medicine Journal, 28(6), 467-471. https://doi.org/10.1136/emj.2009.081869

Gunnell, D., Appleby, L., Arensman, E., Hawton, K., John, A., Kapur, N., et al. (2020). Suicide risk and prevention during the COVID-19 pandemic. The Lancet Psychiatry, 7(6), 468-471. https://doi.org/10.1016/S2215-0366(20)30171-1

House, A., \& Owens, D. (2020). General hospital services in the UK for adults presenting after self-harm: little evidence of progress in the past 25 years. The British Journal of Psychiatry, 1-2. https://doi.org/10.1192/bjp.2020.85

Howick J, Moscrop A, Mebius A, Fanshawe TR, Lewith G, Bishop FL, Mistiaen P, Roberts NW, Dieninyte E, Hu XY, \& Aveyard P. (2018). Effects of empathic and positive communication in healthcare consultations: a systematic review and meta-analysis. Journal of the Royal Society of Medicine,111(7):240-52. https://doi.org/10.1177/0141076818769477 
Hunter C, Chantler K, Kapur N, \& Cooper J. (2013). Service user perspectives on psychosocial assessment following self-harm and its impact on further help-seeking: a qualitative study. Journal of affective disorders, 145(3):315-23.

https://doi.org/10.1016/j.jad.2012.08.009

Kapur, N., Murphy, E., Cooper, J., Bergen, H., Hawton, K., Simkin, S., ... \& Owens, D. (2008). Psychosocial assessment following self-harm: results from the multi-centre monitoring of self-harm project. Journal of affective disorders, 106(3), 285-293. https://doi.org/10.1016/j.jad.2007.07.010

MacDonald S, Sampson C, Turley R, Biddle L, Ring N, Begley R, \& Evans R. (2020). Patients' Experiences of Emergency Hospital Care Following Self-Harm: Systematic Review and Thematic Synthesis of Qualitative Research. Qualitative Health Research, 30(3):471-85. https://doi.org/10.1177/1049732319886566

Morphet, J., Innes, K., Munro, I., O’Brien, A., Gaskin, C. J., Reed, F., \& Kudinoff, T. (2012). Managing people with mental health presentations in emergency departments-A service exploration of the issues surrounding responsiveness from a mental health care consumer and carer perspective. Australasian Emergency Nursing Journal, 15(3), 148-155.

https://doi.org/10.1016/j.aenj.2012.05.003

National Institute for Clinical Excellence (2013). Self-harm: the short-term physical and psychological management and secondary prevention of self-harm in primary and secondary care. accessed from: www.nice.org.uk/guidance/qs34.

Office for National Statistics (2019). Suicides in the UK: 2018 registrations. Statistical bulletin. ONS, accessed from:

https://www.ons.gov.uk/peoplepopulationandcommunity/birthsdeathsandmarriages/deaths/bu lletins/suicidesintheunitedkingdom/2018registrations\#main-points

Owens, C., Hansford, L., Sharkey, S., \& Ford, T. (2016). Needs and fears of young people presenting at accident and emergency department following an act of self-harm: secondary analysis of qualitative data. The British Journal of Psychiatry, 208(3), 286-291.

https://doi.org/10.1192/bjp.bp.113.141242

Philippot P, Feldman R, \& Coats E (2003). The role of nonverbal behavior in clinical settings. In: Philippot P, Feldman R, Coats E, editors. Nonverbal Behavior in Clinical Settings. New York, NY: Oxford University Press, pp. 3-1.

Pompili, M., Girardi, P., Ruberto, A., Kotzalidis, G. D., \& Tatarelli, R. (2005). Emergency staff reactions to suicidal and self-harming patients. European Journal of Emergency Medicine, 12(4), 169-178. https://doi.org/10.1097/00063110-200508000-00005

Priebe, S., Conneely, M., McCabe, R., \& Bird, V . (2020). What can clinicians do to improve outcomes across psychiatric treatments: a conceptual review of non-specific components. Epidemiology and Psychiatric Sciences, 29. https://doi.org/10.1017/S2045796019000428 
Taylor, T. L., Hawton, K., Fortune, S., \& Kapur, N. (2009). Attitudes towards clinical services among people who self-harm: systematic review. The British Journal of Psychiatry, 194(2), 104-110. https://doi.org/10.1192/bjp.bp.107.046425

Thomas, K. C., Owino, H., Ansari, S., Adams, L., Cyr, J. M., Gaynes, B. N., \& Glickman, S. W. (2018). Patient-centered values and experiences with emergency department and mental health crisis care. Administration and Policy in Mental Health and Mental Health Services Research, 45(4), 611-622. https://doi.org/10.1007/s10488-018-0849-y

Vatne, M., \& Nåden, D. (2014). Patients' experiences in the aftermath of suicidal crises. Nursing ethics, 21(2), 163-175. https://doi.org/10.1177/0969733013493218 